\title{
September 2017 Arizona Thoracic Society Notes
}

The September 2017 Arizona Thoracic Society meeting was held on Wednesday, September 27, 2017 at the HonorHealth Rehabilitation Hospital beginning at 6:30 PM. This was a dinner meeting with case presentations. There were 16 in attendance representing the pulmonary, critical care, sleep, and radiology communities.

There was a discussion of the Tobacco 21 bill which had been introduced the last session in the Arizona State Legislature. Since it seems likely that the bill will be reintroduced, the Arizona Thoracic Society will support the bill in the future. Dr. Rick Robbins announced that the SWJPCC has applied to be included in PubMed. In addition, Dr. Robbins was assigned the task of tracking down the campaign contributions to congressional members from the tobacco PAC before the next election.

There were 7 case presentations:

1. Ashley L. Garrett, MD, pulmonary fellow at Mayo, presented an elderly man with insulin-dependent diabetes who felt he had inhaled a pill. He takes multiple medications and was unsure which pill he might have inhaled. Since the inhalation, he was bothered by coughing. His chest x-ray was normal. Bronchoscopy revealed severe left lower lobe bronchitis. No pill fragments were seen. He was managed conservatively and his coughing has nearly resolved. A discussion of pill aspiration ensued with an article published in Chest forming the basis for discussion (1).

2. Paul Conomos, M.D. presented a case of a 57-year-old woman who is largely asymptomatic but has had worsening bronchiectasis on serial CT scans since 2006. She is a nonsmoker. The CT scans show typical tree-in-bud bronchiectasis most marked in the right upper lobe but present in scattered areas throughout both right and left lungs. Her pulmonary function tests showed mild-moderate obstruction. Bronchoscopy times three with bronchoalveolar lavage and cultures has been unrevealing. Alpha-1 antitrypsin levels and esophageal $\mathrm{pH}$ monitoring were normal. Sweat chloride was equivocal at 44 and 50 millimoles per liter. Gene sequencing was recommended but too expensive for the patient ( $\$ 2500$, her copay $\$ 900)$. Discussion focused on whether further work up should be done and whether treatment was necessary. Most felt the work up was fairly comprehensive and that treatment was probably not indicated since she was not symptomatic.

3. Dr. Conomos also presented a second case of an 18-year-old from the Congo who presented with a chronic cough and hemoptysis. PPD was reported by the patient as negative. Physical examination was unremarkable. Chest $x$-ray showed a right lower lobe mass and thoracic CT scan showed right lower lobe $(R L L)$ bronchiectasis with a question of a foreign body. Bronchoscopy showed obstruction in the lateral subsegment of the RLL with a mass with what appeared to be a stone. The patient was referred to thoracic surgery but 
returned 6 days later with fever and pleuritic chest pain. Chest x-ray showed RLL pneumonia. The patient underwent a RLL lobectomy. A foreign body was present. In retrospect, his mother recalled him inhaling a super glue cap when he was 7 or 8 years old. He was doing well post-operatively.

4. Dr. Gerald Schwartzberg presented 3 cases. The first was 43-year-old woman who developed erythema nodosum after a month history of sharp pleuritic chest pain and multiple other systemic complaints. Her eosinophil count was $13 \%$ and cocci serologies were weakly positive. Discussion centered on treatment. Most favored treatment although it was agreed that data supporting treatment was lacking.

5. Dr. Schwartzberg presented a second case of 75-year-old woman with mild COPD on albuterol only. She was a smoker and complained of a cough productive of green sputum. Chest $\mathrm{x}$-ray revealed a large left mass with mucoid impaction. Bronchoscopy revealed hyphae with $45^{\circ}$ branches typical of Aspergillus on biopsy. Thoracic CT scan showed bronchiectasis. An IgE was suggested. Several were suspicious of lung cancer and suggested a needle biopsy of the mass.

6. The last of Dr. Schwartzberg's cases was a 92-year-old man who was found to have a polyp on upper Gl endoscopy and a chest x-ray which showed a mass. Biopsies of both stained positive for melanin and were consistent with malignant melanoma. He was referred to oncology. Discussion centered on whether he should receive treatment.

7. Dr. Lewis Wesselius presented a 67-year-old man with a right neck mass found in 2015. Biopsy revealed a high-grade sarcomatoid cancer. At that time a CT/PET of the chest was negative. About 6 months later a CT/PET revealed new areas of tracer accumulation within the liver. His chemotherapy was switched to ipilimumab and nivolumab. A repeat CT/PET showed symmetric bilateral mediastinal lymphadenopathy. An endobronchial bronchial ultrasound (EBUS) biopsy of the nodes showed noncaseating granuloma consistent with sarcoidosis. He was begun on corticosteroids and nodes and liver lesions resolved on CT/PET. Discussion centered on sarcoidosis induced by these newer checkpoint inhibitors. It was speculated that drug-induced sarcoidosis might be observed more commonly as these agents are more frequently used $(2,3)$.

There being no further business, the meeting was adjourned about 8 PM. The next meeting will be in Phoenix on Wednesday, November 15, 2017 at 6:30 PM at HonorHealth Rehabilitation Hospital.

Richard A. Robbins, MD

\section{References}

1. Kinsey CM, Folch E, Majid A, Channick CL. Evaluation and management of pill aspiration: case discussion and review of the literature. Chest. 2013 Jun;143(6):1791-5. [CrossRef] [PubMed] 
2. Reuss JE, Kunk PR, Stowman AM, Gru AA, Slingluff CL Jr, Gaughan EM. Sarcoidosis in the setting of combination ipilimumab and nivolumab immunotherapy: a case report \& review of the literature. J Immunother Cancer. 2016 Dec 20;4:94. [CrossRef] [PubMed]

3. Danlos FX, Pagès $C$, Baroudjian $B$, et al. Nivolumab-induced sarcoid-like granulomatous reaction in a patient with advanced melanoma. Chest. 2016 May;149(5):e133-6. [CrossRef] [PubMed] 